\title{
Real Time Nuclear Magnetic Resonance Detection of Fumarase Activity using Parahydrogen-Hyperpolarized $\left[1-{ }^{13} \mathrm{C}\right]$ fumarate
}

\author{
James Eills ${ }^{a, *}$, Eleonora Cavallari ${ }^{b}$, Carla Carrera $^{\mathrm{c}}$, Dmitry Budker ${ }^{\mathrm{a}, \mathrm{d}}$, Silvio Aime ${ }^{\mathrm{b}}$, and Francesca Reineri ${ }^{\mathrm{b}}$
}

a) Helmholtz Institute, Johannes Gutenberg University, Mainz, Germany, b) Dept. of Molecular Biotechnology and Health Sciences, University of Torino, Torino, Italy c) Institute of Biostructures and Bioimaging, National Research Council of Italy, Torino, Italy d) Department of Physics, University of California, Berkeley, U.S.A., ( $\left.{ }^{*}\right)$ Corresponding author. Email: eills@uni-mainz.de

\begin{abstract}
Hyperpolarized fumarate can be used as a probe of real-time metabolism in vivo, using carbon-13 magnetic resonance imaging. Dissolution dynamic nuclear polarization is commonly used to produce hyperpolarized fumarate, but a cheaper and faster alternative is to produce hyperpolarized fumarate via PHIP (parahydrogen induced polarization). In this work we trans-hydrogenate $\left[1-{ }^{13} \mathrm{C}\right]$ acetylene dicarboxylate with para-enriched hydrogen using a commercially available $\mathrm{Ru}$ catalyst in water to produce hyperpolarized $\left[1{ }^{13} \mathrm{C}\right]$ fumarate. We show that fumarate is produced in $89 \%$ yield, with succinate as a side product in $11 \%$ yield. The proton polarization is converted into ${ }^{13} \mathrm{C}$ magnetization using a constant adiabaticity field cycle, and a polarization level of $25 \%$ is achieved using $86 \%$ para-enriched hydrogen gas. We inject the hyperpolarized $\left[1-{ }^{13} \mathrm{C}\right]$ fumarate into cell suspensions and track the metabolism. This work opens the path to greatly accelerated preclinical studies using fumarate as a biomarker.
\end{abstract}

\section{Introduction}

MRI (magnetic resonance imaging) is a powerful, noninvasive medical technique, but has limitations due to the intrinsic low sensitivity. To overcome this problem, it is possible to hyperpolarize the nuclear spins, a procedure that has been shown to produce signal enhancements of biomolecules up to $10^{5}$ at high field $[1,2]$. Unfortunately, the hyperpolarization decays in (typically) tens of seconds due to spin relaxation. Despite this temporal limitation, it is possible to observe real-time cellular metabolism in vitro and in vivo after injection of hyperpolarized metabolites [3-6].

One promising candidate metabolite is $\left[1,4-{ }^{13} \mathrm{C}_{2}\right]$ fumarate, which is converted to $\left[1,4-{ }^{13} \mathrm{C}_{2}\right]$ malate by the enzyme fumarase in one step of the citric acid cycle. Fumarate transport through the cell membrane is a relatively slow process, and so significant formation of malate is only observed in regions of necrotic cells where fumarate has access to the enzyme. This change in chemical identity can be observed in carbon-13 MRI via CSI (chemical shift imaging), and has been shown to be a sensitive marker of cell necrosis [79]. The technique is currently being assessed for application in clinical trials [4] which opens exciting new possibilities for imaging of cell necrosis and diagnostics of therapeutic response $[9,10]$. All in vivo experiments to date have used $\left[1,4-{ }^{13} \mathrm{C}_{2}\right]$ fumarate polarized via dDNP (dissolution dynamic nuclear polarization) [11]. There are three significant drawbacks to this technique: (1) it is prohibitively expensive, (2) complex cryogenic equipment is required to reach $1.4 \mathrm{~K}$, and (3) the hyperpolarized material is delivered in batch-mode, with tens of minutes of preparation time required between sample deliveries.
PHIP (parahydrogen induced polarization) is an alternative hyperpolarization modality which involves chemical addition of hydrogen gas enriched in the para spin isomer to a substrate molecule [2,12]. PHIP is approximately 1-2 orders of magnitude less expensive than dDNP, and can produce hyperpolarized products with a much higher duty cycle because it only requires chemical reaction with hydrogen gas. However, the application of this powerful tool to in vivo metabolic studies is hampered by the limited number of metabolites that can be hyperpolarized by means of parahydrogen addition to an unsaturated precursor of the target molecule [13-16]. Hyperpolarized $\left[1-{ }^{13} \mathrm{C}\right]$ fumarate has

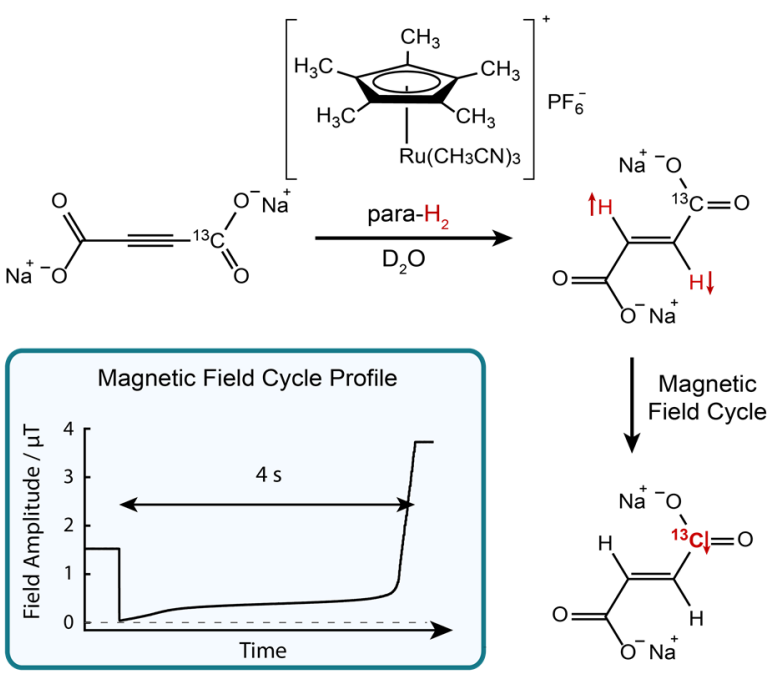

Figure 1: Reaction scheme showing the chemical addition of para-enriched hydrogen to an unsaturated $\left[1-{ }^{13} \mathrm{C}\right]$ acetylene dicarboxylate precursor, to yield $\left[1-{ }^{13} \mathrm{C}\right]$ fumarate. The proton singlet order is then transformed into ${ }^{13} \mathrm{C}$ magnetization by applying a constant-adiabaticity magnetic field cycle [18], optimized for the $\left[1-{ }^{13} \mathrm{C}\right]$ fumarate $J$-couplings. The fieldcycling profile is shown in the inset. 
been recently obtained from the pairwise addition of parahydrogen to $\left[1-{ }^{13} \mathrm{C}\right]$ acetylene dicarboxylate, thanks to the use of a trans-hydrogenative Ru-based catalyst (Fig. 1) [17]. This represents a significant development, because the most widely used hydrogenative PHIP catalyst (a Rh-based complex) leads to cishydrogenation of substrates, and its use here would lead to toxic maleate being obtained [18]. However, in order to progress to meaningful metabolic application, it is crucial to improve the fumarate polarization level and yield of the hydrogenation reaction.

In this work the unsaturated precursor molecule $\left[1-{ }^{13} \mathrm{C}\right]$ acetylene dicarboxylate at a concentration of 50 $\mathrm{mM}$ in $\mathrm{D}_{2} \mathrm{O}$ was completely hydrogenated with parahydrogen ( $86 \%$ enriched) at $80^{\circ} \mathrm{C}$ and a pressure of 9.6 bar in a high-pressure NMR tube to produce $\left[1-{ }^{13} \mathrm{C}\right.$ ]fumarate. $\mathrm{D}_{2} \mathrm{O}$ is used (rather than $\mathrm{H}_{2} \mathrm{O}$ ) to extend the lifetime of the spin order. After reaction, the protons are hyperpolarized in a nonmagnetic 'singlet state' [19]. In our experiment, the proton singlet order was transformed into hyperpolarized magnetization on the $1^{1-13} \mathrm{C}$ spin by subjecting the sample to a magnetic field cycle [20,21]. Here, a 'constant adiabaticity' [22] fieldcycling profile was used. The chemical reaction and magnetic field cycle profile are shown in Fig. 1.

\section{Results}

To measure the polarization achieved on the hydrogenated substrate, the NMR tube used for the reaction was placed into the high field magnet immediately after the field cycle. A comparison between the hyperpolarized and thermal equilibrium spectra is shown in Fig. 2. The resulting signal enhancement factor was measured to be 20,300 at $14.1 \mathrm{~T}$, corresponding to a polarization level of $25 \%$. The ratio of peak integrals between fumarate and succinate indicates $89 \%$ fumarate yield ( $45 \mathrm{mM})$, and $11 \%$ succinate yield $(5 \mathrm{mM})$,

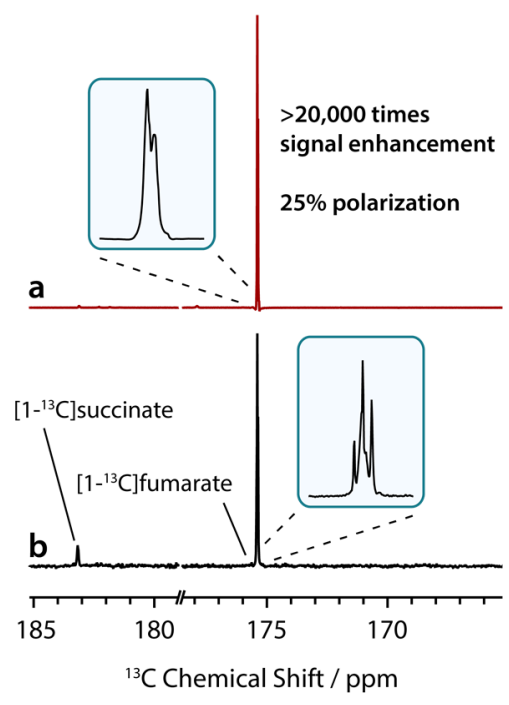

Figure 2: ${ }^{13} \mathrm{C}$ NMR spectra shown with $0.5 \mathrm{~Hz}$ line broadening acquired without proton decoupling. The region 178.5 to 179 ppm contained a pulse artifact and has been cut for clarity. a) A hyperpolarized spectrum of the reaction sample acquired after hydrogenation with parahydrogen, magnetic field cycle, and transport to high field. The hyperpolarized spectrum was acquired with a single transient after applying a pulse with a flip-angle of $5^{\circ}$. b) A thermal equilibrium spectrum of the reaction sample acquired after the hyperpolarized signals had fully relaxed. The thermal equilibrium spectrum was acquired with 512 transients using $90^{\circ}$ flip-angle pulses, and has been vertically expanded by a factor of 4 .

and there is no detectable unreacted starting material. We have set an upper bound on the concentration of unreacted starting material at $<0.4 \mathrm{mM}$.

To study PHIP-polarized fumarate as a probe of cellular necrosis, experiments were performed in which the reaction mixture containing hyperpolarized $\left[1-{ }^{13} \mathrm{C}\right]$ fumarate was added to cell suspensions. Three experiments were performed; one in which the cell media contained 10 million lysed EL-4 tumour cells, one with 10 million intact (healthy) EL-4 tumour cells, and one with

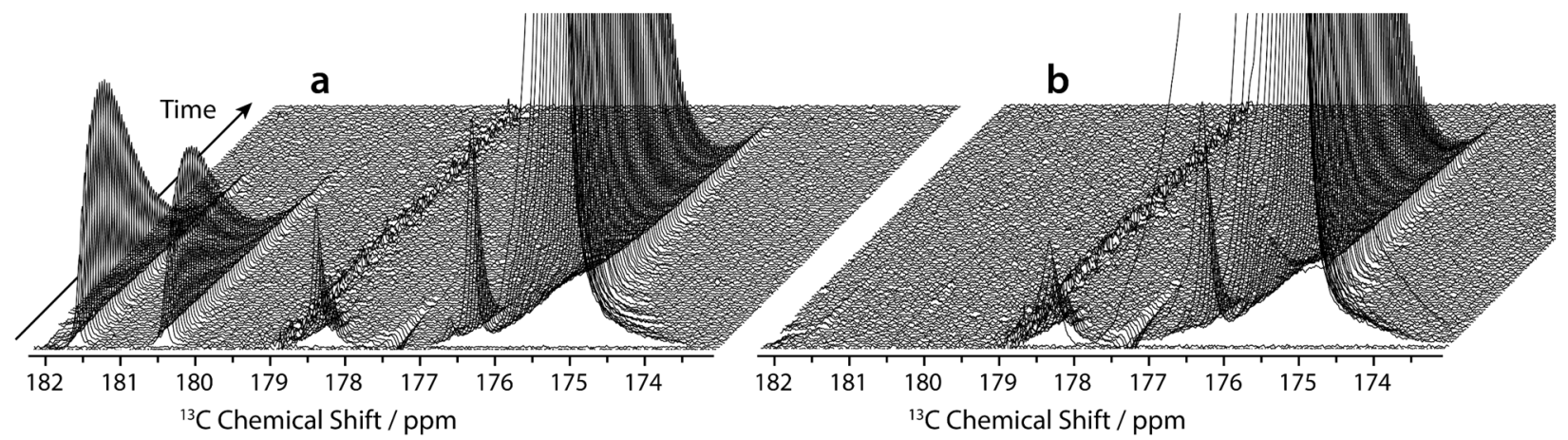

Figure 3: Hyperpolarized ${ }^{13} \mathrm{C}$ NMR spectra shown with $0.5 \mathrm{~Hz}$ line broadening acquired without proton decoupling. Both datasets were acquired on reaction samples after hydrogenation with parahydrogen, magnetic field cycle, and injection into a suspension of (a) lysed, and (b) intact (live) EL-4 tumour cells, at high field. Spectra were acquired every $2 \mathrm{~s}$ using $15^{\circ}$ flip-angle pulses. The peaks at $175.4 \mathrm{ppm}$ correspond to $\left[1-{ }^{13} \mathrm{C}\right]$ fumarate, and the peaks in (a) at 180.5 and $181.7 \mathrm{ppm}$ correspond to $\left[4-{ }^{13} \mathrm{C}\right] \mathrm{malate}$ and [1${ }^{13} \mathrm{C}$ ]malate, respectively. The rapidly relaxing peaks at 176.4 and $178.5 \mathrm{ppm}$ are thought to be low concentration catalyst sideproducts. 
no cells in the media. In these experiments, after perfusion of the $\left[1-{ }^{13} \mathrm{C}\right]$ fumarate containing solution through the cells suspension, the ${ }^{13} \mathrm{C}$ NMR signal was acquired every $2 \mathrm{~s}$ using $15^{\circ}$ flip-angle pulses, so as to probe the metabolism over time without significantly perturbing the magnetization.

From the control experiment without cells in the media, and after applying a correction for signal decay due to continual if pulsing [23], the fumarate $T_{1}$ under our experimental conditions was measured to be $27.5 \mathrm{~s}$.

A stack plot of the ${ }^{13} \mathrm{C}$ NMR spectra for the two experiments carried out in the presence of cells is shown in Fig. 3. In the experiment with intact cells, fumarate transport across the cell membrane is slow relative to $T_{1}$, so no malate signal is detected. In the experiment with lysed cells, the fumarate has access to the enzyme and reacts with water to form malate. The asymmetry of this molecule means the ${ }^{13} \mathrm{C}$ spin is distributed between the 1 and 4 positions, and $\left[1-{ }^{13} \mathrm{C}\right]$ malate and $\left[4-{ }^{13} \mathrm{C}\right]$ malate signals are observed at 181.7 and $180.5 \mathrm{ppm}$, respectively. The signal reaches a maximum at $18-20 \mathrm{~s}$ after the injection, and decays due to $T_{1}$ relaxation and continual of pulsing. The $\left[1-{ }^{13} \mathrm{C}\right]$ fumarate signal is at $175.4 \mathrm{ppm}$, and is significantly larger than the malate signals.

The fumarate and malate peak integrals are plotted in Fig. 4, and a fit to the data is obtained using a kinetic model that only includes fumarate conversion to malate, and relaxation of both species:

$$
F \stackrel{k}{\rightarrow} M .
$$

We can write the following differential equations:

$$
\begin{aligned}
& \frac{d F(t)}{d t}=-k F(t)-\frac{1}{T_{\mathrm{x}}} F(t), \\
& \frac{d M(t)}{d t}=k F(t)-\frac{1}{T_{\mathrm{x}}} M(t),
\end{aligned}
$$

where $F(t)$ is the time-dependent concentration of hyperpolarized $\left[1-{ }^{13} \mathrm{C}\right]$ fumarate, $M(t)$ is the timedependent concentration of hyperpolarized $\left[1-{ }^{13} \mathrm{C}\right]$ malate and $\left[4-{ }^{13} \mathrm{C}\right]$ malate, and $k$ is the forward reaction-rate constant. In this model, for simplicity we assume that both species relax with the same time constant $T_{\mathrm{x}}$, which is a combination of $T_{1}$ and the signal decay due to successive pulses. $T_{\mathrm{x}}$ is known from the $\left[1-{ }^{13} \mathrm{C}\right]$ fumarate measurement discussed above, and the rate constant $k$ was treated as a fitting parameter. This model yielded $k=1.62 \pm 0.01 \times 10^{-3} \mathrm{~s}^{-1}$. Including a rate constant for the reverse reaction did not improve the quality of the fit. We note that the measured rate constant is higher than has been previously reported [9]. This may

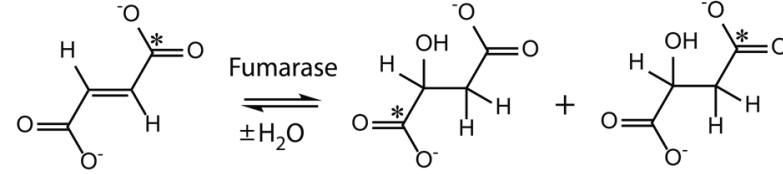

$$
\left[1-{ }^{13} \mathrm{C}\right] \text { fumarate } \quad\left[1-{ }^{13} \mathrm{C}\right] \text { malate } \quad\left[4-{ }^{13} \mathrm{C}\right] \text { malate }
$$

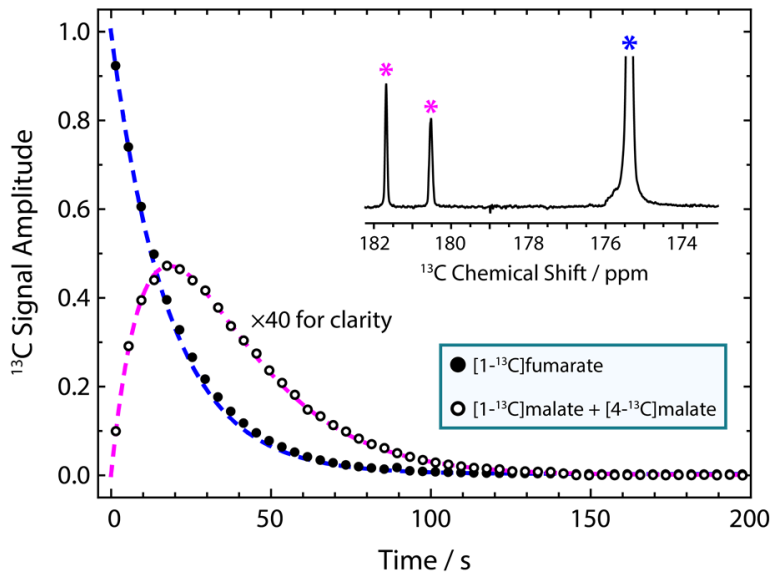

Figure 4: Top: The enzymatic conversion of $\left[1-{ }^{13} \mathrm{C}\right]$ fumarate into both $\left[1-{ }^{13} \mathrm{C}\right]$ malate and $\left[4-{ }^{13} \mathrm{C}\right]$ malate. Bottom: Flux of ${ }^{13} \mathrm{C}-$ label between fumarate and malate in a suspension of lysed EL-4 tumour cells. The filled black dots represent integrals of $\left[1-{ }^{13} \mathrm{C}\right]$ fumarate and hollow black dots represent integrals of [1$\left.{ }^{13} \mathrm{C}\right]$ malate $+\left[4-{ }^{13} \mathrm{C}\right]$ malate signals. The signals have been normalized, with 1 corresponding to the initial $\left[1-{ }^{13} \mathrm{C}\right]$ fumarate signal. In both datasets, every other datapoint has been dropped for clarity, and the malate signal amplitude has been magnified by a factor of 40 . The error bars are contained within the data points. The inset spectrum shows a single acquisition from the dataset. Fits to the data are shown by dashed blue (fumarate) and magenta (malate) lines.

be due to a difference in the experimental conditions; specifically, a higher cell concentration was used in this work.

\section{Discussion}

To explain the dramatic increase in ${ }^{13} \mathrm{C}$ polarization over the original demonstration $\left(2 \%{ }^{13} \mathrm{C}\right.$ polarization, using $50 \%$ para-enriched hydrogen) [17], we consider the key experimental differences. In the previously reported experiments, the hydrogenation reaction took $30 \mathrm{~s}$ and was performed by bubbling hydrogen gas into a reaction solution at $50{ }^{\circ} \mathrm{C}$ in the high field NMR magnet. In the present case, the reaction was faster $(10 \mathrm{~s})$, and was carried out by shaking the sample at $80^{\circ} \mathrm{C}$ in Earth's magnetic field $(\sim 50 \mu \mathrm{T})$. Firstly, by using a shorter reaction time in this work, there was less time for spin relaxation to occur. Secondly, it is possible that parahydrogen singlet order is lost due to singlet/triplet mixing occurring on the reaction intermediates [24]; this destructive process is likely to be more efficient at high magnetic field due to larger chemical shift differences between the parahydrogen protons on the reaction intermediates. Thirdly, longer hydrogenation times may lead to partial degradation of the catalyst, with formation 
of metal-containing species which may enhance the rate of parahydrogen relaxation. It should also be considered that the reaction solution in the previous work was likely not at $50^{\circ} \mathrm{C}$ as expected, because the bubbling of roomtemperature hydrogen gas would lower the reaction temperature.

In previous studies employing dDNP as the polarization technique, the doubly ${ }^{13} \mathrm{C}$-labelled isotopomer $\left[1,4-{ }^{13} \mathrm{C}_{2}\right]$ fumarate was used. Here, the $\left[1-{ }^{13} \mathrm{C}\right]$ isotopomer was used, which means the maximum achievable ${ }^{13} \mathrm{C}$ signal is lower by a factor of 2 . There are unitary bounds on the achievable polarization transfer from proton singlet order into ${ }^{13} \mathrm{C}$ magnetization, which depend on the symmetry of the spin system [25]. Assuming an initial state of pure proton singlet order, the maximum possible transfer into $\mathrm{X}$-spin $\left({ }^{13} \mathrm{C}\right)$ polarization for the $\left[1^{13} \mathrm{C}\right]$ isotopomer, an $A A^{\prime} X$ spin system (Pople notation), is 1 , corresponding to $100 \%{ }^{13} \mathrm{C}$ polarization. However, for the $\left[1,4-{ }^{13} \mathrm{C}_{2}\right]$ isotopomer, an $\mathrm{AA}^{\prime} X X^{\prime}$ spin system, the ${ }^{13} \mathrm{C}$ polarization of each is limited to $50 \%$. This therefore gives the same observable ${ }^{13} \mathrm{C}$ signal enhancement as using the $\left[1^{13} \mathrm{C}\right]$ isotopomer. This limitation cannot be overcome without further reducing the symmetry of the spin system. It could still be beneficial to use the $\left[1,4-{ }^{13} C_{2}\right]$ isotopomer, because the relaxation properties of the spins should differ based on the symmetry, which might yield higher overall signal enhancements.

The NMR measurements were carried out at 14.1 T, and the fumarate ${ }^{1-{ }^{13}} \mathrm{C} \quad T_{1}$ was $27.5 \mathrm{~s}$. The dominant relaxation mechanism is chemical shift anisotropy [26], which means a markedly longer ${ }^{13} \mathrm{C} T_{1}$ is expected by working at lower magnetic field. This is an exciting perspective for future applications of $\left[1-{ }^{13} \mathrm{C}\right]$ fumarate in clinical imaging scanners, which typically employ field strengths of 1-3 T.

In conclusion, we have demonstrated that PHIP allows for the production of highly polarized $\left[1-{ }^{13} \mathrm{C}\right]$ fumarate at a concentration of $45 \mathrm{mM}$ in aqueous solution. Using relatively inexpensive and easily operated PHIP equipment, $a{ }^{13} \mathrm{C}$ polarization level of $25 \%$ was observed on $\left[1-{ }^{13} \mathrm{C}\right]$ fumarate. For the reaction, we used $86 \%$ paraenriched hydrogen gas, meaning the polarization level could be increased to $31 \%$ by switching to $100 \%$ paraenriched hydrogen. Following the perfusion of lysed cells with the fumarate solution, metabolic transformation into malate was observed. This work paves the way for accelerated preclinical studies with hyperpolarized fumarate.

\section{Acknowledgements}

This project has received funding from the European Union's Horizon 2020 research and innovation programme under the Marie Skłodowska-Curie Grant Agreement No. 766402, and Compagnia di San Paolo (Athenaeum Research 2016, n. CST0164550). The authors would like to thank Bogdan Rodin for calculating the constant adiabaticity field-cycling profile, and Christian Bengs for invaluable help running experiments.

\section{Materials and Methods}

\section{Cell culture}

The EL-4 murine lymphoma cell line was purchased from American Type Culture Collection (ATCC) and grown in Dulbecco's Modified Eagle's Media supplemented with 10\% Fetal Bovine Serum and $1 \%$ Penicillin Streptomycin. Cells were cultured in Corning $\mathrm{T}-175$ flasks at $37^{\circ} \mathrm{C}$ in a humidified atmosphere containing $5 \% \mathrm{CO}_{2}$. Cultures were maintained at $10^{5}-10^{6}$ cells $/ \mathrm{ml}$ by addition of fresh media every 2 days.

\section{Hydrogenation and polarization transfer}

All the chemicals were purchased from Sigma Aldrich. In a solution of $50 \mathrm{mM}$ disodium $\left[1-{ }^{13} \mathrm{C}\right]$ acetylene dicarboxylate and $100 \mathrm{mM}$ sodium sulphite in $\mathrm{D}_{2} \mathrm{O}$, the ruthenium catalyst $\left[\mathrm{RuCp} *\left(\mathrm{CH}_{3} \mathrm{CN}\right)_{3}\right] \mathrm{PF}_{6}$ was dissolved by gentle heating and sonication. The solution was filtered using a PTFE syringe filter (Whatman UNIFLO) with $0.22 \mu \mathrm{m}$ diameter pores, with a final catalyst concentration of $8 \mathrm{mM} .300 \mu \mathrm{l}$ of the filtered solution was frozen in a $5 \mathrm{~mm}$ pressurisable NMR tube equipped with a J. Young valve. The tube was held in a liquid nitrogen bath $(77 \mathrm{~K})$ and pressurized with 2.1 bar of para-enriched hydrogen (86\% enriched). The sample was kept at $77 \mathrm{~K}$ to prevent any chemical reaction before the start of the hyperpolarization experiment.

To initiate the hydrogenation reaction, the NMR tube was heated in a hot water bath at $80^{\circ} \mathrm{C}$ for $30 \mathrm{~s}$ which melted the sample and raised the internal pressure to 9.6 bar, then shaken vigorously for $10 \mathrm{~s}$. The NMR tube was opened to release the parahydrogen pressure and immediately placed in a magnetic shield (Aspect Imaging, Shoham, Israel) for the application of a magnetic field cycle. Magnetic fields were applied using a solenoid coil controlled by a Keysight Technologies 33220A $20 \mathrm{MHz}$ waveform generator (Keysight Technologies, Santa Rosa, U.S.). The field was initially set at $1.5 \mathrm{mT}$, dropped to $50 \mathrm{nT}$ in less than $1 \mathrm{~ms}$, then increased adiabatically to $3.7 \mu \mathrm{T}$ in $4 \mathrm{~s}$ (see Fig. 1). The sample was then removed from the shield for NMR experiments. 
NMR experiments were performed at $14.1 \mathrm{~T}$ in a $5 \mathrm{~mm}$ BBO probe using a Bruker AVANCE console. In the experiment to obtain the ${ }^{13} \mathrm{C}$ polarization level, spectra were acquired of the hyperpolarized reaction mixture every $2 \mathrm{~s}$ using $5^{\circ}$ flip-angle pulses. After this, a thermal equilibrium spectrum of the sample was acquired with 512 transients, using $90^{\circ}$ flip-angle pulses separated by $90 \mathrm{~s}$ intervals.

\section{Cell suspension experiments}

After hydrogenation of the $300 \mu \mathrm{l}$ sample and magnetic field cycle, the hyperpolarized reaction mixture was collected in a syringe containing $150 \mu$ l of acidic buffer solution (HEPES $144 \mathrm{mM}, \mathrm{pH} 5.5$ ) to produce a $450 \mu \mathrm{l}$ solution with physiological osmolarity (290 $\pm 2 \mathrm{mOsm})$ and optimal $\mathrm{pH}(7.6 \pm 0.2)$ for the enzyme activity. $200 \mu \mathrm{l}$ of the hyperpolarized reaction mixture was injected into a $5 \mathrm{~mm}$ NMR tube via syringe through PTFE tubing (1.6 $\mathrm{mm}$ O.D., $0.8 \mathrm{~mm}$ I.D.) in the high field NMR magnet. The NMR tube contained $200 \mu \mathrm{l}$ of culture media at $310 \mathrm{~K}$.

The EL-4 tumour cells were counted using a Burker chamber, centrifuged in the growth media for $5 \mathrm{~min}$ at $125 \mathrm{x}$ g-force, and the supernatant was removed. $10.0 \pm$ 0.1 million cells were suspended in $200 \mu \mathrm{l}$ of culture media and transferred into a $5 \mathrm{~mm}$ NMR test tube for experiments. For experiments on lysed cells, this cell suspension was double freeze-thawed in liquid nitrogen. The NMR tube (containing either lysed or intact cells) was then placed in the NMR magnet and equilibrated at $310 \mathrm{~K}$. At the end of each experiment the number of cells was quantified using a Bradford protein assay with a specific calibration line, and was measured to be $10 \pm$ 0.1 million in all experiments.

\section{References}

[1] Nikolaou, P.; Goodson, B. M.; and Chekmenev, E. Y.; NMR hyperpolarization techniques for biomedicine, Chem. Eur. J. 2015, 21, 3156-3166

[2] Green, R. A.; Adams, R. W.; Duckett, S. B.; Mewis, R. E.; Williamson, D. C.; Green, G. G. R.; The theory and practice of hyperpolarization in magnetic resonance using parahydrogen, $P$. Nucl. Mag. Res. Sp. 2012, 67, 1-48

[3] Wang, Z. J.; Ohliger, M. A.; Larson, P. E. Z.; Gordon, J. W.; Bok, R. A.; Slater, J.; Villanueva-Meyer, J. E.; Hess, C. P.; Kurhanewicz, J.; Vigneron, D. B.; Hyperpolarized ${ }^{13} \mathrm{C}$ MRI: State of the Art and Future Directions, Radiology 2019, 291, 273-284

[4] Kurhanewicz, J.; Vigneron, D. B.; ArdenkjaerLarsen, J. H.; et al.; Hyperpolarized ${ }^{13} \mathrm{C}$ MRI: Path to Clinical Translation in Oncology, Neoplasia 2019, 21, 1-16
[5] Nelson, S. J.; Kurhanewicz, J.; Vigneron, D. B.; et al.; Metabolic imaging of patients with prostate cancer using hyperpolarized $\left[1{ }^{13} \mathrm{C}\right]$ pyruvate, Sci. Transl. Med. 2013, 5, 198ra108-198ra108

[6] Cavallari, E.; Carrera, C.; Sorge, M.; Bonne, G.; Muchir, A.; Aime, S.; Reineri, F.; The ${ }^{13} \mathrm{C}$ hyperpolarized pyruvate generated by parahydrogen detects the response of the heart to altered metabolism in real time, Sci. Rep. 2018, 8, 8366-8375

[7] Clatworthy, M. R.; Kettunen, M. I.; Hu, D.-E.; et al.; Magnetic resonance imaging with hyperpolarized $\left[1,4-{ }^{13} \mathrm{C}_{2}\right]$ fumarate allows detection of early renal acute tubular necrosis, Proc. Natl. Acad. Sci. U.S.A. 2012, 109, 13374-13379

[8] Miller, J. J.; Lau, A. Z.; Nielsen, P. M.; McMullenKlein, G.; Lewis, A. J.; Jespersen, N. R.; Ball, V.; Gallagher, F. A.; Carr, C. A.; Laustsen, C.; Bøtker H. E.; Tyler D. J.; Schroeder, M. A.; Hyperpolarized $\left[1,4-{ }^{13} \mathrm{C}_{2}\right]$ Fumarate Enables Magnetic ResonanceBased Imaging of Myocardial Necrosis, JACC Cardiovasc. Imaging 2018, 11, 1594-1606

[9] Gallagher, F. A.; Kettunen, M. I.; Hu D.-E.; et al.; Production of hyperpolarized $\left[1,4-{ }^{13} \mathrm{C}_{2}\right]$ malate from $\left[1,4-{ }^{13} \mathrm{C}_{2}\right]$ fumarate is a marker of cell necrosis and treatment response in tumors, Proc. Natl. Acad. Sci. U.S.A. 2009, 106, 19801-19806

[10]Mignion, L.; Dutta, P.; Martinez, G. V.; Foroutan, P.; Gillies R. J.; Jordan, B. F.; Monitoring chemotherapeutic response by hyperpolarized ${ }^{13} \mathrm{C}$ fumarate MRS and diffusion MRI, Cancer Res. 2014, 74, 686-694

[11] Ardenkjaer-Larsen, J. H.; On the present and future of dissolution-DNP, J. Magn. Reson. 2016, 264, 3-12

[12] Bowers, C. R.; Weitekamp, D. P.; Parahydrogen and synthesis allow dramatically enhanced nuclear alignment, J. Am. Chem. Soc. 1987, 109, 5541-5542 [13] Cavallari, E.; Carrera, C.; Reineri, F.; ParaHydrogen Hyperpolarized Substrates for Molecular Imaging Studies, Isr. J. Chem. 2017, 57, 833-842

[14] Chekmenev, E. Y.; Hövener, J.; Norton, V. A.; Harris, K.; Batchelder, L. S.; Bhattacharya, P.; Ross, B. D.; Weitekamp, D. P. PASADENA Hyperpolarization of Succinic Acid for MRI and NMR Spectroscopy. J. Am. Chem. Soc. 2008, 130, 4212-4213

[15] Shchepin, R. V; Coffey, A. M.; Waddell, K. W.; Chekmenev, E. Y. PASADENA Hyperpolarized 13C Phospholactate. J. Am. Chem. Soc. 2012, 134, 3957-3960

[16] Reineri, F.; Boi, T.; Aime, S. ParaHydrogen Induced Polarization of $13 \mathrm{C}$ Carboxylate Resonance in Acetate and Pyruvate. Nat. Commun. 2015, 6, 5858 [17] Ripka, B.; Eills, J.; Kourilova, H.; Leutzsch, M.; Levitt, M. H.; Münnemann, K.; Hyperpolarized fumarate via parahydrogen, Chem. Commun. 2018, 54, 
12246-12249

[18] Eills, J.; Stevanato, G.; Bengs, C.; Glöggler, S.;

Elliott, S. J.; Alonso-Valdesueiro, J.; Pileio, G.; Levitt, M. H.; Singlet order conversion and parahydrogeninduced hyperpolarization of ${ }^{13} \mathrm{C}$ nuclei in nearequivalent spin systems, J. Magn. Reson. 2017, 274, 163-172

[19] Levitt, M. H.; Singlet nuclear magnetic resonance, Annu. Rev. Phys. Chem. 2012, 63, 89-105

[20] Jóhannesson, H.; Axelsson, O.; Karlsson, M. Transfer of Para-Hydrogen Spin Order into Polarization by Diabatic Field Cycling. Comptes Rendus Phys.

2004, 5, 315-324

[21] Cavallari, E.; Carrera, C.; Boi, T.; Aime, S.; Reineri, F.; Effects of magnetic field cycle on the polarization transfer from parahydrogen to heteronuclei through long-range J-couplings, J. Phys. Chem. B 2015, 119, 10035-10041

[22] Rodin, B. A.; Sheberstov, K. F.; Kiryutin, A. S.; et al.; Constant-adiabaticity radiofrequency pulses for generating long-lived singlet spin states in NMR, J. Chem. Phys. 2019, 150, 064201

[23] Balzan, R.; Fernandes, L.; Pidial, L.; Comment, A.; Tavitian, B.; Vasos, P. R.; Pyruvate cellular uptake and enzymatic conversion probed by dissolution DNP-NMR: the impact of overexpressed membrane transporters, Magn. Reson. Chem. 2017, 55, 579-583

[24] Kating, P.; Wandelt, A.; Selke, R.; Bargon, J.; Nuclear singlet/triplet mixing during hydrogenations with parahydrogen: an in situ NMR method to investigate catalytic reaction mechanisms and their kinetics. 2 . Homogeneous hydrogenation of 1,4-dihydro-1,4epoxynaphthalene using different rhodium catalysts, $J$. Phys. Chem. 1993, 97, 13313-13317

[25] Levitt, M. H.; Symmetry constraints on spin dynamics: Application to hyperpolarized NMR, J. Magn. Reson. 2016, 262, 91-99

[26] Eills, J.; Alonso-Valdesueiro, J.; Salazar Marcano, D. E.; da Silva, J. F.; Alom, S.; Rees, G. J.; Hanna, J. V.; Carravetta, M.; Levitt, M. H.; Preservation of nuclear spin order by precipitation, ChemPhysChem 2018, 19, 40-44 